International Journal of Pure and Applied Mathematics

Volume $91 \quad$ No. 4 2014, 421-433

ISSN: 1311-8080 (printed version); ISSN: 1314-3395 (on-line version)

url: http://www.ijpam.eu

doi: http://dx.doi.org/10.12732/ijpam.v91i4.1

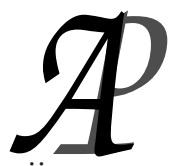

ijpam.eu

\title{
ON SUMMATION OF SUBSERIES OF THE RIEMANN ZETA FUNCTION
}

\author{
Hongwei Chen \\ Department of Mathematics \\ Christopher Newport University \\ Newport News, VA 23606, USA
}

\begin{abstract}
Three classes of subseries of the Riemann zeta function are evaluated in closed form. All the results are expressed in terms of the Riemann zeta function itself and powers of $\pi$. By using the Euler totient function, we prove that these three classes are unique subseries that exhibit this kind of closed form.
\end{abstract}

AMS Subject Classification: 11M06, 11M35, 11B68, 33B10

Key Words: Riemann zeta function, Hurwitz zeta function, Bernoulli polynomials, reflection sums, derivatives of the cotangent.

\section{Introduction}

Let $\zeta(s)$ be the Riemann zeta function defined by

$$
\zeta(s):=\sum_{n=1}^{\infty} \frac{1}{n^{s}}
$$

The series converges for all $s>1$. There are many formulas related the subseries

Received: October 8, 2013

(c) 2014 Academic Publications, Ltd. url: www.acadpubl.eu 
of the Riemann zeta function

$$
\zeta(a, b, s):=\sum_{k=0}^{\infty} \frac{1}{(a k+b)^{s}}, \quad(a, b, s \in \mathbb{N}, \operatorname{gcd}(a, b)=1)
$$

to $\zeta(s)$ itself, for example, from the well-known expression

$$
\zeta(2,1, s)=\sum_{k=0}^{\infty} \frac{1}{(2 k+1)^{s}}=\left(1-2^{-s}\right) \zeta(s)
$$

to more involved results such as

$$
\begin{aligned}
& \zeta(3,1,3)=\sum_{k=0}^{\infty} \frac{1}{(3 k+1)^{3}}=\frac{1}{2}\left(1-3^{-3}\right) \zeta(3)+\frac{2}{3^{5}} \sqrt{3} \pi^{3}, \\
& \zeta(3,1,5)=\sum_{k=0}^{\infty} \frac{1}{(3 k+1)^{5}}=\frac{1}{2}\left(1-3^{-5}\right) \zeta(5)+\frac{2}{3^{7}} \sqrt{3} \pi^{5} .
\end{aligned}
$$

Motivated by such intriguing results, it is natural for us to ask

Q1. When does a given series $\zeta(a, b, s)$ have a closed form?

Q2. Is the closed form always a linear combination of $\pi^{s}$ and $\zeta(s)$ ?

Notice that these series are all subseries of the Riemann zeta function. Appealing to the polylogarithm of Lewin, $\operatorname{Li}_{s}(z)$ (see [3, p.189] or [4, p.611]) defined by

$$
\operatorname{Li}_{s}(z):=\sum_{k=1}^{\infty} \frac{z^{k}}{k^{s}}
$$

the series multisection formula (see [2, Theorem 10.1, p.110]) yields, for example,

$$
\zeta(3,1,3)=\sum_{k=0}^{\infty} \frac{1}{(3 k+1)^{3}}=\frac{1}{3}\left(\operatorname{Li}_{3}(1)+\frac{1}{\omega} \operatorname{Li}_{3}(\omega)+\frac{1}{\omega^{2}} \operatorname{Li}_{3}\left(\omega^{2}\right)\right)
$$

where $\omega=e^{2 \pi i / 3}$, the primitive cubic root. However, due to the limited knowledge of $\operatorname{Li}_{s}(z)$ for complex arguments (see [3]), we can't recover (3) from (6).

Using the contour integral, Zucker and Robertson [7] evaluated some Dirichlet $L$-series exactly, which are alternating series of (1). The main results are summarized in the Appendix A of [1]. In this paper, we present another method to study $\zeta(a, b, s)$ in closed form. We will show that the affirmative answers to Q1 and Q2 only hold for $a=3,4,6$ and $s$ odd. Our approach is in purely Eulerian style, and our all manipulation of series and appearance of identities have the familiar Eulerian flair. 


\section{Proof of (3)}

Before we proceed to develop the closed form for $\zeta(a, b, s)$, we give an elementary proof of (3), and will see that the proof of the extensions follows by analogy.

First, inspired by Euler's infinite product formula

$$
\zeta(s)=\prod_{p \text { prime }}\left(1-p^{-s}\right)^{-1},
$$

we find that

$$
\begin{aligned}
\zeta(3)\left(1-3^{-3}\right) & =1+\frac{1}{2^{3}}+\frac{1}{4^{3}}+\frac{1}{5^{3}}+\frac{1}{7^{3}}+\frac{1}{8^{3}}+\frac{1}{10^{3}}+\frac{1}{11^{3}}+\cdots \\
& =\left(1+\frac{1}{4^{3}}+\frac{1}{7^{3}}+\cdots\right)+\left(\frac{1}{2^{3}}+\frac{1}{5^{3}}+\frac{1}{8^{3}}+\cdots\right) \\
& =\sum_{k=0}^{\infty} \frac{1}{(3 k+1)^{3}}+\sum_{k=0}^{\infty} \frac{1}{(3 k+2)^{3}} .
\end{aligned}
$$

On the other hand, recall that Hurwitz zeta function (see [4, Chapter 25.11, p.607]), which is defined by

$$
\zeta(s, a):=\sum_{k=0}^{\infty} \frac{1}{(k+a)^{s}} .
$$

First we will need a lemma that converts the series into an integral.

Lemma 1. For $s>1$ we have

$$
\zeta(s, a)=\frac{1}{\Gamma(s)} \int_{0}^{\infty} \frac{x^{s-1} e^{-a x}}{1-e^{-x}} d x,
$$

where $\Gamma$ is the Euler gamma function defined by

$$
\Gamma(s)=\int_{0}^{\infty} t^{s-1} e^{-t} d t
$$

Proof. Note that

$$
\frac{1}{1-e^{-x}}=\sum_{k=0}^{\infty} e^{-k x}
$$

Therefore,

$$
\int_{0}^{\infty} \frac{x^{s-1} e^{-a x}}{1-e^{-x}} d x=\sum_{k=0}^{\infty} \int_{0}^{\infty} x^{s-1} e^{-(k+a) x} d x
$$


The change of variable $t=(k+a) x$ yields

$$
\int_{0}^{\infty} \frac{x^{s-1} e^{-a x}}{1-e^{-x}} d x=\sum_{k=0}^{\infty} \frac{1}{(k+a)^{s}} \int_{0}^{\infty} t^{s-1} e^{-t} d t=\zeta(s, a) \Gamma(s),
$$

which is equivalent to (8).

Next, in terms of the integral representation (8), we establish a lemma connecting the reflection sum of the Hurwitz zeta function:

Lemma 2. For $0<\mu<1, n \in \mathbb{N}$ we have

$$
\zeta(n, \mu)-(-1)^{n-1} \zeta(n, 1-\mu)=\frac{(-1)^{n-1} \pi}{(n-1) !}(\cot (\mu \pi))^{(n-1)},
$$

where $f^{(k)}$ denotes the $k$-th derivative.

Proof. Applying (8) we see that

$$
\begin{aligned}
\zeta(n, \mu)-(-1)^{n-1} \zeta(n, 1-\mu) & \\
= & \frac{1}{(n-1) !} \int_{0}^{\infty} \frac{x^{n-1}\left(e^{-\mu x}-(-1)^{n-1} e^{-(1-\mu) x}\right)}{1-e^{-x}} d x
\end{aligned}
$$

Via the parametric differentiation, this can be related to

$$
f(\mu)=\int_{0}^{\infty} \frac{e^{-\mu x}-e^{-(1-\mu) x}}{1-e^{-x}} d x .
$$

In terms of (9), we have

$$
\begin{aligned}
f(\mu) & =\int_{0}^{\infty} \frac{e^{-\mu x}}{1-e^{-x}} d x-\int_{0}^{\infty} \frac{e^{-(1-\mu) x}}{1-e^{-x}} d x \\
& =\sum_{k=0}^{\infty} \frac{1}{k+\mu}-\sum_{k=0}^{\infty} \frac{1}{k+1-\mu} \\
& =\sum_{k=0}^{\infty} \frac{1}{k+\mu}+\sum_{k=0}^{\infty} \frac{1}{-(k+1)+\mu} \\
& =\sum_{k=-\infty}^{\infty} \frac{1}{k+\mu} .
\end{aligned}
$$


Appealing to Euler's elegant partial fraction decomposition (see [2, Eq. 6.27, p.68])

$$
\cot x=\sum_{k=-\infty}^{\infty} \frac{1}{k \pi+x}
$$

we find that

$$
f(\mu)=\pi \cot (\mu \pi) .
$$

Taking $(n-1)$ th derivative of $f(\mu)$ gives the desired result $(10)$.

Now, returning to (3), notice that

$$
\sum_{k=0}^{\infty} \frac{1}{(3 k+1)^{3}}-\sum_{k=0}^{\infty} \frac{1}{(3 k+2)^{3}}=\frac{1}{3^{3}}(\zeta(3,1 / 3)-\zeta(3,2 / 3)) .
$$

Putting $n=3$ in (10) yields

$$
\sum_{k=0}^{\infty} \frac{1}{(3 k+1)^{3}}-\sum_{k=0}^{\infty} \frac{1}{(3 k+2)^{3}}=\left.\frac{\pi}{2 ! \cdot 3^{3}}(\cot (\mu \pi))^{\prime \prime}\right|_{\mu=1 / 3}=\frac{4}{3^{5}} \sqrt{3} \pi^{3} \text {. }
$$

In conjunction with (7), this yields (3) as expected. As a byproduct, we also find the closed form for its reflection sum

$$
\sum_{k=0}^{\infty} \frac{1}{(3 k+2)^{3}}=\frac{1}{2}\left(1-3^{-3}\right) \xi(3)-\frac{2}{3^{5}} \sqrt{3} \pi^{3} .
$$

In general, observe that if $n=2 m+1$, applying (10) yields

$$
\begin{aligned}
& \sum_{k=0}^{\infty} \frac{1}{(3 k+1)^{2 m+1}}-\sum_{k=0}^{\infty} \frac{1}{(3 k+2)^{2 m+1}} \\
= & \frac{1}{3^{2 m+1}}(\zeta(2 m+1,1 / 3)-\zeta(2 m+1,2 / 3))=\left.\frac{\pi}{3^{2 m+1}(2 m) !}(\cot (\mu \pi))^{(2 m)}\right|_{\mu=1 / 3} .
\end{aligned}
$$

Coupling with

$$
\sum_{k=0}^{\infty} \frac{1}{(3 k+1)^{n}}+\sum_{k=0}^{\infty} \frac{1}{(3 k+2)^{n}}=\left(1-3^{-n}\right) \zeta(n)
$$

we obtain 
H. Chen

$$
\begin{aligned}
& \sum_{k=0}^{\infty} \frac{1}{(3 k+1)^{2 m+1}} \\
& \quad=\frac{1}{2}\left(1-3^{-(2 m+1)}\right) \zeta(2 m+1)+\left.\frac{\pi}{2 \cdot 3^{2 m+1}(2 m) !}(\cot (\mu \pi))^{(2 m)}\right|_{\mu=1 / 3}, \\
& \sum_{k=0}^{\infty} \frac{1}{(3 k+2)^{2 m+1}} \\
& \quad=\frac{1}{2}\left(1-3^{-(2 m+1)}\right) \zeta(2 m+1)-\left.\frac{\pi}{2 \cdot 3^{2 m+1}(2 m) !}(\cot (\mu \pi))^{(2 m)}\right|_{\mu=1 / 3} .
\end{aligned}
$$

In particular, setting $m=2$ and using that $\left.(\cot (\mu \pi))^{(4)}\right|_{\mu=1 / 3}=32 \sqrt{3} \pi^{4} / 3$, we arrive at (4).

However, if $n=2 m,(10)$ yields the same equation as (14). In contrast to (2) where $a=2$, we are unable to find the closed form for

$$
\zeta(3,1,2 m)=\sum_{k=0}^{\infty} \frac{1}{(3 k+1)^{2 m}}
$$

in terms of $\zeta(2 m)$ and $\pi^{2 m}$, even for $m=1$.

As we end this section, for rational $\mu$, we show how the values of $\cot (\mu \pi)^{(n)}$ are related to the Bernoulli polynomials - another favorite of Euler. This lemma will enable us to answer Q2.

Lemma 3. If $a, b, n \in \mathbb{N}, 1 \leq b<a$ and $(a, b)=1$, then

$$
\begin{aligned}
& \left.(\cot (\mu \pi))^{(2 n)}\right|_{\mu=b / a}=(-1)^{n-1} \frac{2(2 a \pi)^{2 n}}{2 n+1} \sum_{j=1}^{a} B_{2 n+1}\left(\frac{j}{a}\right) \sin \left(\frac{2 b j \pi}{a}\right), \\
& \left.(\cot (\mu \pi))^{(2 n-1)}\right|_{\mu=b / a}=(-1)^{n} \frac{(2 a \pi)^{2 n-1}}{n} \sum_{j=1}^{a} B_{2 n}\left(\frac{j}{a}\right) \cos \left(\frac{2 b j \pi}{a}\right) .
\end{aligned}
$$

Here $B_{n}(x)$ is the Bernoulli polynomial of degree $n$ (see [4, Chapter 24]).

Proof. The approach is based on another way to evaluate $\zeta(n, \mu) \pm \zeta(n, 1-$ $\mu)$. Notice that

$$
\zeta(n, b / a)=a^{n} \zeta(a, b, n)
$$


Applying the series multisection formula and (5), we have

$$
\zeta(n, b / a)=a^{n-1} \sum_{j=1}^{a} \omega^{-j b} \operatorname{Li}_{n}\left(\omega^{j}\right)
$$

where $\omega=e^{2 \pi i / a}$. Simplification yields

$$
\begin{aligned}
& \zeta(n, b / a)+\zeta(n, 1-b / a)=2 a^{n-1} \sum_{j=1}^{a}\left(\sum_{k=1}^{\infty} \frac{\cos (2 k j \pi / a)}{k^{n}}\right) \cos \left(\frac{2 b j \pi}{a}\right) \\
& \zeta(n, b / a)-\zeta(n, 1-b / a)=2 a^{n-1} \sum_{j=1}^{a}\left(\sum_{k=1}^{\infty} \frac{\sin (2 k j \pi / a)}{k^{n}}\right) \sin \left(\frac{2 b j \pi}{a}\right)
\end{aligned}
$$

The proposed formulas (17) and (18) now follow from the Fourier series for $B_{n}(x)$ (see [4, Eq. 24.8.1 and Eq. 24.8.2, p.592]):

$$
\begin{aligned}
B_{2 n}(x) & =(-1)^{n-1} \frac{2(2 n) !}{(2 \pi)^{2 n}} \sum_{k=1}^{\infty} \frac{\cos (2 k x \pi)}{k^{2 n}}, \\
B_{2 n-1}(x) & =(-1)^{n} \frac{2(2 n-1) !}{(2 \pi)^{2 n-1}} \sum_{k=1}^{\infty} \frac{\sin (2 k x \pi)}{k^{2 n-1}} .
\end{aligned}
$$

When $a=3, b=1$, in view of the well-known fact that $B_{2 m+1}(1)=0$ and the formulas

$$
\begin{aligned}
B_{n}(0) & =(-1)^{n} B_{n}(1)=B_{n}, \quad(\text { see }[4, \text { Eq. 24.4.25, p.590]), } \\
B_{n}(x) & =(-1)^{n} B_{n}(1-x), \quad(\text { see }[4, \text { Eq. 24.4.3, p.589]) } \\
B_{2 m}(1 / 3) & =\frac{1}{2}\left(3^{1-2 m}-1\right) B_{2 m}, \quad(\text { see [4, Eq. 24.4.29, p.590]), }
\end{aligned}
$$

where $B_{n}$ is the Bernoulli number, applying (17) and (18) we have

$$
\begin{aligned}
\left.(\cot (\mu \pi))^{(2 m)}\right|_{\mu=1 / 3} & =(-1)^{m-1} \frac{2 \cdot 6^{2 m} B_{2 m+1}(1 / 3)}{2 m+1} \sqrt{3} \pi^{2 m}, \\
\left.(\cot (\mu \pi))^{(2 m-1)}\right|_{\mu=1 / 3} & =(-1)^{m} \frac{2^{2 m-1}\left(3^{2 m}-1\right)}{2 m} B_{2 m} \pi^{2 m-1} .
\end{aligned}
$$

In conjunction with (14), by (24) we recover another Euler's famous result:

$$
\zeta(2 m)=\frac{1}{1-3^{-2 m}}\left(\sum_{k=0}^{\infty} \frac{1}{(3 k+1)^{2 m}}+\sum_{k=0}^{\infty} \frac{1}{(3 k+2)^{2 m}}\right)
$$




$$
\begin{aligned}
& =\frac{3^{2 m}}{3^{2 m}-1} \frac{1}{3^{2 m}}(\zeta(2 m, 1 / 3)+\zeta(2 m, 2 / 3)) \\
& =-\left.\frac{\pi}{\left(3^{2 m}-1\right)(2 m-1) !}(\cot (\mu \pi))^{(2 m-1)}\right|_{\mu=1 / 3} \\
& =(-1)^{m+1} \frac{2^{2 m-1}}{(2 m) !} B_{2 m} \pi^{2 m} .
\end{aligned}
$$

Furthermore, (23) and (24) enable us to rewrite (15) and (16) as

$$
\begin{aligned}
\sum_{k=0}^{\infty} \frac{1}{(3 k+1)^{2 m+1}=} & \frac{1}{2}\left(1-3^{-(2 m+1)}\right) \zeta(2 m+1) \\
& +(-1)^{m-1} \frac{2^{2 m}}{3 \cdot(2 m+1) !} B_{2 m+1}(1 / 3) \sqrt{3} \pi^{2 m+1} \\
\sum_{k=0}^{\infty} \frac{1}{(3 k+2)^{2 m+1}}= & \frac{1}{2}\left(1-3^{-(2 m+1)}\right) \zeta(2 m+1) \\
& +(-1)^{m} \frac{2^{2 m}}{3 \cdot(2 m+1) !} B_{2 m+1}(1 / 3) \sqrt{3} \pi^{2 m+1}
\end{aligned}
$$

These formulas yield the affirmative answer to Q1 and Q2 for $a=3, s=2 m+1$.

\section{Extensions to $a=4,6$}

Observe that finding the closed form of $\zeta(3,1, n)$ is based on a system of two equations for $\zeta(3,1, n)$ and its reflection sum $\zeta(3,2, n)$. Here, when $n$ is odd, (10) provides one equation, while the decomposition (14) supplies another. Along these lines, in this section we establish closed formulas of $\zeta(a, b, n)$ for $a=4,6$ and $n$ odd. First, similar to (7), we have

$$
\begin{aligned}
\zeta(n)\left(1-2^{-n}\right) & =1+\frac{1}{3^{n}}+\frac{1}{5^{n}}+\frac{1}{7^{n}}+\frac{1}{9^{n}}+\frac{1}{11^{n}}+\frac{1}{13^{n}}+\frac{1}{15^{n}}+\cdots \\
& =\left(1+\frac{1}{5^{n}}+\frac{1}{9^{n}}+\cdots\right)+\left(\frac{1}{3^{n}}+\frac{1}{7^{n}}+\frac{1}{11^{n}}+\cdots\right) \\
& =\sum_{k=0}^{\infty} \frac{1}{(4 k+1)^{n}}+\sum_{k=0}^{\infty} \frac{1}{(4 k+2)^{n}} .
\end{aligned}
$$

On the other hand, using (10), we find that

$$
\sum_{k=0}^{\infty} \frac{1}{(4 k+1)^{n}}-(-1)^{n-1} \sum_{k=0}^{\infty} \frac{1}{(4 k+2)^{n}}=\left.\frac{(-1)^{n-1} \pi}{4^{n}(n-1) !}(\cot (\mu x))^{(n-1)}\right|_{\mu=1 / 4} .
$$


If $n=2 m+1$, solving the coupled system of (27) and (28) yields

$$
\begin{aligned}
\sum_{k=0}^{\infty} \frac{1}{(4 k+1)^{2 m+1}=} & \frac{1}{2}\left(1-2^{-(2 m+1)}\right) \zeta(2 m+1) \\
& +\left.\frac{\pi}{2 \cdot 4^{2 m+1}(2 m) !}(\cot (\mu \pi))^{(2 m)}\right|_{\mu=1 / 4}, \\
\sum_{k=0}^{\infty} \frac{1}{(4 k+3)^{2 m+1}=} & \frac{1}{2}\left(1-2^{-(2 m+1)}\right) \zeta(2 m+1) \\
& -\left.\frac{\pi}{2 \cdot 4^{2 m+1}(2 m) !}(\cot (\mu \pi))^{(2 m)}\right|_{\mu=1 / 4} .
\end{aligned}
$$

By (17) and (21), we have

$$
\begin{aligned}
\left.(\cot (\mu \pi))^{(2 m)}\right|_{\mu=1 / 4} & =(-1)^{m-1} \frac{2 \cdot 8^{2 m}}{2 m+1} \pi^{2 m}\left(B_{2 m+1}(1 / 4)-B_{2 m+1}(3 / 4)\right) \\
& =(-1)^{m-1} \frac{4 \cdot 8^{2 m} B_{2 m+1}(1 / 4)}{2 m+1} \pi^{2 m}
\end{aligned}
$$

This transforms (29) and (30) into

$$
\begin{aligned}
\sum_{k=0}^{\infty} \frac{1}{(4 k+1)^{2 m+1}=} & \frac{1}{2}\left(1-2^{-(2 m+1)}\right) \zeta(2 m+1) \\
& +(-1)^{m-1} \frac{2^{2 m+1}}{4 \cdot(2 m+1) !} B_{2 m+1}(1 / 4) \pi^{2 m+1}, \\
\sum_{k=0}^{\infty} \frac{1}{(4 k+3)^{2 m+1}=} & \frac{1}{2}\left(1-2^{-(2 m+1)}\right) \zeta(2 m+1) \\
& +(-1)^{m} \frac{2^{2 m+1}}{4 \cdot(2 m+1) !} B_{2 m+1}(1 / 4) \pi^{2 m+1} .
\end{aligned}
$$

Therefore, when $a=4, n=2 m+1$, we obtain the positive answers to Q1 and Q2 again. In particular, letting $m=1,2$, we have

$$
\begin{aligned}
\zeta(4,1,3) & \left.=\sum_{k=0}^{\infty} \frac{1}{(4 k+1)^{3}}=\frac{1}{2}\left(1-2^{-3}\right) \zeta 3\right)+\frac{1}{2^{6}} \pi^{3}, \\
\zeta(4,3,3) & =\sum_{k=0}^{\infty} \frac{1}{(4 k+3)^{3}}=\frac{1}{2}\left(1-2^{-3}\right) \zeta(3)-\frac{1}{2^{6}} \pi^{3}, \\
\zeta(4,1,5) & =\sum_{k=0}^{\infty} \frac{1}{(4 k+1)^{5}}=\frac{1}{2}\left(1-2^{-5}\right) \zeta(5)+\frac{5}{3 \cdot 2^{10}} \pi^{5},
\end{aligned}
$$




$$
\zeta(4,3,3)=\sum_{k=0}^{\infty} \frac{1}{(4 k+3)^{5}}=\frac{1}{2}\left(1-2^{-5}\right) \zeta(3)-\frac{5}{3 \cdot 2^{10}} \pi^{5} .
$$

On the other hand, if $n$ is even, for example, we have

$$
\zeta(4,1,2)=\sum_{k=0}^{\infty} \frac{1}{(4 k+1)^{2}}=\frac{1}{2}\left(1-2^{-2}\right) \zeta(2)+\frac{1}{2} G,
$$

where $G$ is the well-known Catalan number, and so Q2 does not valid. Moreover, we are unable to find the closed form for $\zeta(4,1,2 m)$ for any $m>1$.

We now turn to find the closed form for $\zeta(a, b, n)$ when $a=6$. Applying (10) yields

$$
\sum_{k=0}^{\infty} \frac{1}{(6 k+1)^{n}}-(-1)^{n-1} \sum_{k=0}^{\infty} \frac{1}{(6 k+5)^{n}}=\left.\frac{(-1)^{n-1} \pi}{6^{n}(n-1) !}(\cot (\mu \pi))^{(n-1)}\right|_{\mu=1 / 6} .
$$

On the other hand, stemming from Euler's infinite product formula again gives

$$
\begin{aligned}
\zeta(n)\left(1-2^{-n}\right)\left(1-3^{-n}\right) & =1+\frac{1}{5^{n}}+\frac{1}{7^{n}}+\frac{1}{11^{n}}+\frac{1}{13^{n}}+\frac{1}{17^{n}}+\cdots \\
& =\left(1+\frac{1}{7^{n}}+\frac{1}{13^{n}}+\cdots\right)+\left(\frac{1}{5^{n}}+\frac{1}{11^{n}}+\frac{1}{17^{n}}+\cdots\right) \\
& =\sum_{k=0}^{\infty} \frac{1}{(6 k+1)^{n}}+\sum_{k=0}^{\infty} \frac{1}{(6 k+5)^{n}} .
\end{aligned}
$$

When $n=2 m+1$, combining (38) and (39) we have

$$
\begin{aligned}
\sum_{k=0}^{\infty} \frac{1}{(6 k+1)^{2 m+1}=} & \frac{1}{2}\left(1-2^{-(2 m+1)}\right)\left(1-3^{-(2 m+1)}\right) \zeta(2 m+1) \\
& +\left.\frac{\pi}{2 \cdot 6^{2 m+1}(2 m) !}(\cot (\mu \pi))^{(2 m)}\right|_{\mu=1 / 6}, \\
\sum_{k=0}^{\infty} \frac{1}{(6 k+5)^{2 m+1}}= & \frac{1}{2}\left(1-2^{-(2 m+1)}\right)\left(1-3^{-(2 m+1)}\right) \zeta(2 m+1) \\
& -\left.\frac{\pi}{2 \cdot 6^{2 m+1}(2 m) !}(\cot (\mu \pi))^{(2 m)}\right|_{\mu=1 / 6} .
\end{aligned}
$$

Applying (17) and (21) once more we have

$$
\left.(\cot (\mu \pi))^{(2 m)}\right|_{\mu=1 / 6}=(-1)^{m-1} \frac{2 \cdot 12^{2 m} \sqrt{3}}{2 m+1}\left(B_{2 m+1}(1 / 6)+B_{2 m+1}(1 / 3)\right) \pi^{2 m} .
$$


Setting $m=2$ in the Raabe formula (see [4, Eq. 24.4.18, p.590])

$$
B_{n}(x)=\frac{1}{m^{n}} \sum_{k=0}^{m-1} B_{n}\left(x+\frac{k}{m}\right)
$$

gives

$$
B_{2 m+1}(1 / 6)=\left(1+2^{-2 m}\right) B_{2 m+1}(1 / 3),
$$

and thus

$$
\left.(\cot (\mu \pi))^{(2 m)}\right|_{\mu=1 / 6}=(-1)^{m-1} \frac{2 \cdot 12^{2 m} \sqrt{3}}{2 m+1}\left(2+2^{-2 m}\right) B_{2 m+1}(1 / 3) \pi^{2 m} .
$$

This converts (40) and (41) into

$$
\begin{aligned}
\sum_{k=0}^{\infty} \frac{1}{(6 k+1)^{2 m+1}=} & \frac{1}{2}\left(1-2^{-(2 m+1)}\right)\left(1-3^{-(2 m+1)}\right) \zeta(2 m+1) \\
& +(-1)^{m-1} \frac{\left(1+2^{2 m+1}\right)}{6 \cdot(2 m+1) !} B_{2 m+1}(1 / 3) \sqrt{3} \pi^{2 m+1} \\
\sum_{k=0}^{\infty} \frac{1}{(6 k+5)^{2 m+1}}= & \frac{1}{2}\left(1-2^{-(2 m+1)}\right)\left(1-3^{-(2 m+1)}\right) \zeta(2 m+1) \\
& +(-1)^{m} \frac{\left(1+2^{2 m+1}\right)}{6 \cdot(2 m+1) !} B_{2 m+1}(1 / 3) \sqrt{3} \pi^{2 m+1}
\end{aligned}
$$

These yield a positive answer to Q1 and Q2 again. In particular, if $m=1,2$, we have

$$
\begin{aligned}
& \sum_{k=0}^{\infty} \frac{1}{(6 k+1)^{3}}=\frac{1}{2}\left(1-2^{-3}\right)\left(1-3^{-3}\right) \zeta(3)+\frac{1}{2^{2} \cdot 3^{3}} \sqrt{3} \pi^{3} \\
& \sum_{k=0}^{\infty} \frac{1}{(6 k+1)^{5}}=\frac{1}{2}\left(1-2^{-5}\right)\left(1-3^{-5}\right) \zeta(5)+\frac{11}{2^{4} \cdot 3^{6}} \sqrt{3} \pi^{5}
\end{aligned}
$$

The last one was recently proposed as the Monthly Problem 11715 [5].

\section{Uniqueness}

In this section, we show that $\zeta(a, b, n)$ admitting a closed form in terms of $\pi^{n}$ and $\zeta(n)$ only occurs for $a=3,4,5$ and $n$ odd. 
For Q1 and Q2, (37) offers a counterexample when $n$ is even. Thus, we assume that $n$ is odd in what follows. The reflection formula (10) and Lemma 3 demonstrate that $\zeta(a, b, n)-\zeta(a, a-b, n)$ is related to the sine and cosine at rational multiples of $\pi$. To obtain a closed form for $\zeta(a, b, n)$ alone, we need to have another approach to $\operatorname{sum} \zeta(a, b, n)+\zeta(a, a-b, n)$ in terms of $\zeta(n)$. This is equivalent to decomposing $\zeta(n)$ as a sum of $\zeta(a, b, n)$ and $\zeta(a, a-b, n)$. We show this occurs only for $a=3,4,6$. To this end, we recall another tool from Euler. Let $\phi(a)$ be the Euler totient function, i.e., $\phi(a)$ equals the number of positive integers less than $a$ that are relatively prime to $a$. Observe that

$$
\zeta(a, b, n)+\zeta(a, a-b, n)=\sum_{k=0}^{\infty}\left(\frac{1}{(a k+b)^{n}}+\frac{1}{(a k+(a-b))^{n}}\right)=c \zeta(n)
$$

if and only if $\phi(a)=2$. Thus, the desired result follows from the simple fact:

$$
\phi(a)=2 \Longleftrightarrow a=3,4,6 .
$$

As an illustrative example, by (10) we have

$$
\zeta(12,5,3)-\zeta(12,7,3)=\frac{7-4 \sqrt{3}}{3^{3} \cdot 4^{2}} \pi^{3} .
$$

However, since $\phi(12)=4$, using (39), the decomposition of $\zeta(3)$ becomes

$$
\left(1-2^{-3}\right)\left(1-3^{-3}\right) \zeta(3)=\zeta(12,1,3)+\zeta(12,5,3)+\zeta(12,7,3)+\zeta(12,11,3),
$$

instead of $\zeta(12,5,3)+\zeta(12,7,3)$ as expected. So we can't find $\zeta(12,5,3)$ in closed form alone.

Investigating further, the problem of determining the rational numbers $b / a$ such that the sine and cosine values at $b \pi / a$ are radicals was considered by Gauss $([7])$. He associated this problem to constructible regular polygon by compass and ruler, and proved that $a$ must be of the form

$$
a=2^{k} p_{1} p_{2} \cdots p_{l}
$$

where $k \in \mathbb{N}$ and $p_{i}$ are distinct Fermat primes (a prime of the form $2^{2^{\mathrm{n}}}+1$ ). In conjunction with (10) and Lemma 3, we see that Q2 is true only for $a=3,4,6$ again.

Based on Euler's idea and tools, we have found a class of closed forms for $\zeta(a, b, n)$. It is interesting to notice that today we have resources not available to Euler. For example, Mathematica. It is easy to verify our results (3), (4), (33) - (36), (44) and (45) via Mathematica. However, to evaluate (46), Mathematica only returns

-(PolyGamma $[2,5 / 12] / 3456)+\operatorname{PolyGamma}[2,7 / 12] / 3456$

It is hard to see how to reduce this answer to match the result in (46). 


\section{References}

[1] J. M. Borwein, I. J. Zucker and J. Boersma, The evaluation of character Euler double sums, Ramanujan Journal, 15 (2008), 377-405

[2] H. Chen, Excursions in Classical Analysis, Mathematical Association of America, Washington, DC (2010).

[3] L. Lewin, Polylogarithms and Associated Functions, North Holland, New York (1981).

[4] F. W. J. Olver et.al (eds), NIST Handbook of Mathematical Functions, National Institute of Standards and Technology, U.S. Department of Commence \& Cambridge University Press, New York (2010).

[5] M. Stofka, Problem 11715, American Mathematical Monthly, 120 (2013), 569.

[6] E. W. Weisstein, Trigonometry Angles, From MathWorld, A Wolfram Web Resource, Available at http://mathworld.wolfram.com/TrigonometryAngles.html.

[7] I. J. Zucker and M. M. Robertson, Some properties of Dirichlet L-series, J. Phys. A: Math. Gen., 9 (1976), 1207-1214. 
